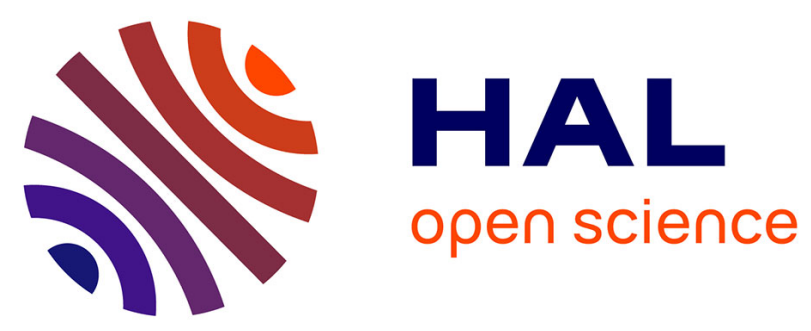

\title{
Une borne routière de l'empereur Nerva à Saintes
}

\author{
Louis Maurin, Francis Tassaux
}

\section{To cite this version:}

Louis Maurin, Francis Tassaux. Une borne routière de l'empereur Nerva à Saintes. Gallia - Fouilles et monuments archéologiques en France métropolitaine, 1979, 37 (2), pp.263-270. 10.3406/galia.1979.1605 . hal-01781741

\section{HAL Id: hal-01781741 \\ https://hal.science/hal-01781741}

Submitted on 4 Feb 2020

HAL is a multi-disciplinary open access archive for the deposit and dissemination of scientific research documents, whether they are published or not. The documents may come from teaching and research institutions in France or abroad, or from public or private research centers.
L'archive ouverte pluridisciplinaire HAL, est destinée au dépôt et à la diffusion de documents scientifiques de niveau recherche, publiés ou non, émanant des établissements d'enseignement et de recherche français ou étrangers, des laboratoires publics ou privés.

\section{(ㅇ)(1) $\$$}

Distributed under a Creative Commons Attribution - NonCommercial - NoDerivatives| 4.0 


\title{
UNE BORNE ROUTIÈRE DE L'EMPEREUR NERVA A SAINTES (Charente-Maritime)
}

\author{
par Louis MAURIN ef Francis TASSAUX
}

A l'automne 1956 une borno routière a été mise au jour à Bellivel, à l'extrémité septentrionale de la commune de saintes. Cette trouvaille n'a été portée à notre connaissance que le 19 juillet 1977. Informé de l'intérìt que présentait ce document. le propriétaire en fil, don sur le champ au IIusée archéologique de, Saintes ${ }^{1}$.

La borne a été découverte lors d'un labour effectué dans la parcelle 18:3, section $A B$ du plan cadastral, à l'emplacement approximatif indiqué sur la fig. 1. Elle était peut-être a l'origine taillée en deux parlies (socle et fût) dans le calcaire. Seule la partie supérieure a été découverte; elle avait été sciée dans le sens de la hauteur à une époque inconnue, de façon à oblenir deux blocs rigoureusement identiques, soit deux demi-cylindres de $89 \mathrm{~cm}$ de hauteur et $59 \mathrm{~cm}$ de diamètre à la base, la partie supérieure de chacun d'eux étant très usée (fig. 2).

Chacun des deux blocs présente, à la base, une cavité en forme de trone de còne renversé, à la panse légèrement arrondie et à l'extrémité adoucie, avec un léger étranglement tout près de l'orifice, si bien que cette cavité a, en somme,

1 Jusqu'en 1977, le propriétaire ctait M. Hervaud, que nous remercions ici de son aimable accucil et de sa générosité. la forme du corps d'une amphore renversée. Le diametre de l'orifice est de $21 \mathrm{~cm}$ et la hauteur de la cavilé de $50 \mathrm{~cm}$. Cette cavité a été creusée avec un grand soin, ses parois sont parfaitement lisses et polies; l'exacte adaptation de chaque partie creusée des deux demi-cylindres semble montrer que cette cavité avait été pratiquée avant que la pierre ne fût sciée verticalement. Nous ignorons sa raison d'être. On peut hésiter à y voir une mortaise destinée à l'ancrage des parties supérieure et inférieure de la borne ćtant donné la forme, les dimensions, le lissage des parois de cette cavité.

sur chacun des deux demi-cylindres se trouve la moitié d'une inscription profondément gravée dans le calcaire à l'intérieur d'un cadre mouluré : dimensions extérieures du cadre : $85 \times 78 \mathrm{~cm}$; largeur de la moulure : $5 \mathrm{~cm}$. On lit dans le champ épigraphique $(71,5 \times 67,5 \mathrm{~cm})$ la titulature de l'empereur Nerva (fig. 2 is 4 ).

$\begin{array}{lr} & \text { Lellres de: } \\ \text { IMP n ERVA } & 95 \mathrm{~mm} \\ \text { CAESARAVG } & 73 \mathrm{~mm} \\ \text { PONTIfEXMAX } & 70 \mathrm{~mm} \\ \text { VMVSTRIBVNIC } & 70 \mathrm{~mm} \\ \text { POTESTATECOSIII } & 70 \mathrm{~mm} \\ \text { P P P } & 115 \mathrm{~mm}\end{array}$

(Les lettres en minuscules manquent sur la pierre, par usure ou cassure, et sont restituées.) 


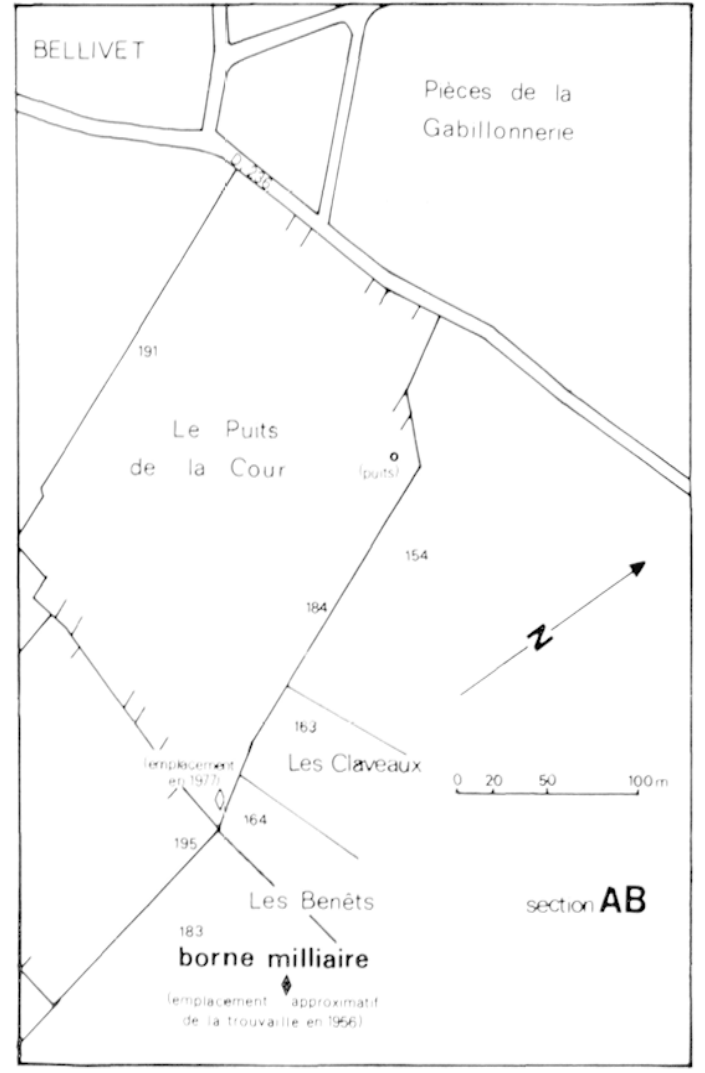

I Silualion de la borne de Bullixel dapris le plan callasimal.

Imp(eralor) [Nerva / Caes[a]r Aug(uslus) / pionlifiex max / umus , l ribunic(ia) / poles $[$ lale, co(n)s(ul) (ler), I p(ater) p(atriae).

L'empereur Nerva a revêtu son troisieme consulat le premier janvier 97 et sa seconde puissance tribunice le 18 septembre 97. I.'inscription de Bellivel a éte gravée entre ces deux dates.

L'usure de la pierre ne cache pas la belle qualité des capitales et le soin de la gravure. Le lapicide a civié les abréviations, sauf pour Aug., qui est de rigle, et, a la derniere ligne, pour le titre de Pere de la Palrie. Noter l'archaïsme maxumus.

Il manque iei une indication capitale, celle de la distance, qui devait consister en un nombre seul ou accompagné éventuellement de l'unité de mesure et du nom du capul viae, du point de départ de la route (ici Mediolanum, sans doute abrégé en MEDj. Il est lien possible que le nombre ail, eté employe seul, si l'on se réfere a la borne des Babinols, lieu-rlit de la commune de siant-Ciers-sur-Cironde, pris de la limile presume entre les Santons et les Biluriges Vivisques. qui date de la méme époque et dont l'errilure el le libelle sont tres proches de celle de Bellivet.

$$
.
$$

L'interet de la borne de Bellivet réside d'abord dans sa dale. Eslant donné la faible durée de son regne, les bornes routieres de Verva sont tris rares dans les (iaules ${ }^{3}$; or relle de Bellivet conduit à altribuer à une initiative de l'administration de ce prince une réforme introduile en Aquitaine dans la gestion des roules.

Ia horne des Babinots a souleve des discussions passionnées ent re spécialistes. Etaitelle rédigée en milles, suivant l'usage romain appliqué depuis le début de l'Empire? (ou bien, dejai, en lieues. suivant la mesure gauloises qui ful genéralise sous les severes, mais dont l'emploi officiel est bien attesté en Aquitaine dis les regnes des Antoninst. Jullian ne pouvail se résoudre a restiluer ici la mention de lieues. llirschfeld el, de maniore plus calegorique. Walsers. croient an contraire que sur la borne

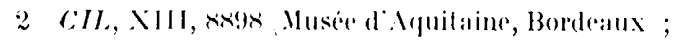
celte borme a de dresseresous le regne de Trajan, entre le 10 dee. 97 el le lo dece 9s, si Trajan porte bien la denxienue puissance lribunice sur la pierre, comme on le croit commumiment. Sinon, entre la mort de Verva ilebut de 9x et la fin de log, date a laquelle Trajan prit le surnom de llacicus, absent sur l'epigraphe. Voir L. VAlExs, Borleath, 2009 ans d'Ilisloire, Bordeanx, $1972, n^{\circ} 66$, p. $7 x$. Comme celle de Bellivet, celle borme a de tromsere seien en dere dans le sens vertical. l.a distance ny est indique que par le sioul chiffre XXVil.

3 lill, Xlll, Mas, sint-Marcel, Meurthe-etMoselle et 91.16 Coblence ; pent-être aussi P. Wun-

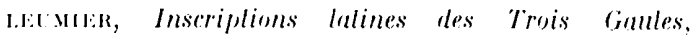

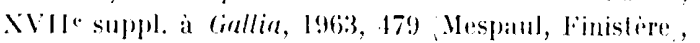

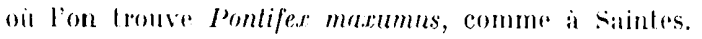

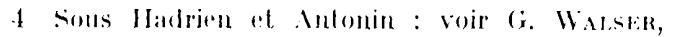
Meilen und Lengen, dams Epigraphica, XXXI, 1969,

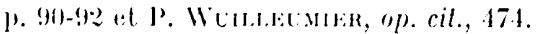

5 1. Judas, Inscriplions romaines de Bordeant,

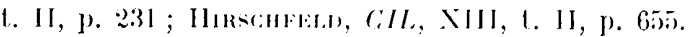
G. WALSER, op. cit., p. Xi. 


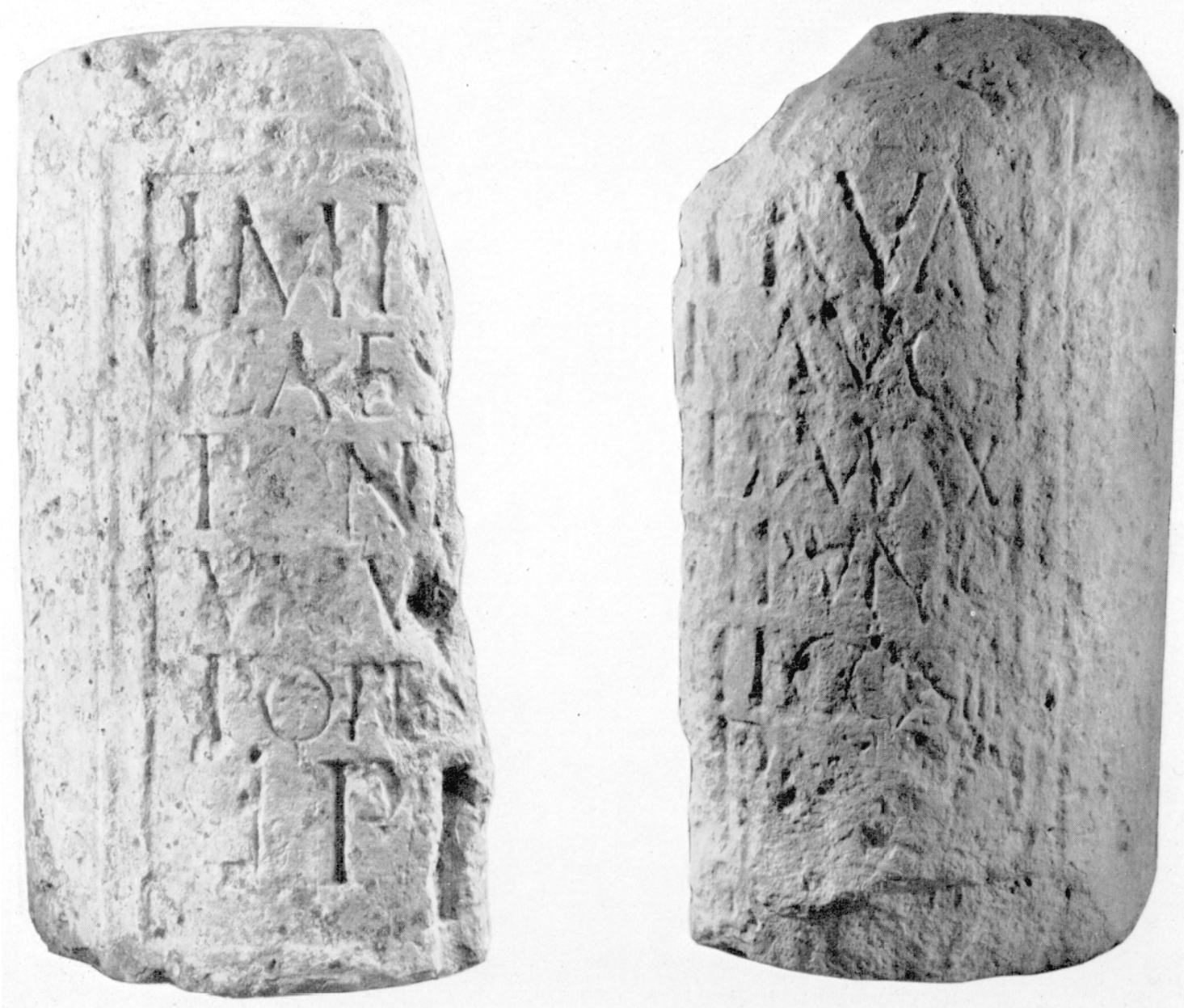

2 La borne de Bellivet.

des Babinots la distance dait exprimée en lieues, ar la pierre a bien été découverte a XXVII lieues (chiffre inscrit sur le bloc) de saintes et, a peu de chose pres, autant de Bordeaux, res deux villes ayant seules pu, en l'occurrence olre les tètes de voies de référence. Walser en tire des conclusions sur l'ormanisation et l'évolution du systeme roulier en Aquilaine, en Gaule, et ailleurs.

Ia numérotation en lieues sur la borne de Bellivet est tris probable, si l'on tient comple d'une part de sa parentio aver celle des Babinots, et d'aulre part d'un document assez récemment, sinnale, decouvert a lssoudun Indre encore dans l'ancienne Aquilaine. Il s'agit d'une borne portant la titulature de Trajan Cos $I I I$, datée de 100 ap. J.-C. A la derniere ligne on lit $A$ (aricum) $L$ (eugas) $X X I I^{6}$. Cette pierre paraît donc contirmer de façon indiscutable que c'est en Aquitaine, dans les années 97-100, que fut inaugurée une nourelle mesure officielle des distances routières, la lieue, empruntée a la tradition ef sans doute à l'usage indigenes. Cetle mutation. qui semble atre restée longtemps limitée à l'Aquitaine. doit traduire limplantation de nouvelles st ructures administratives pour la gestion des routes impériales el, pense-t-on, les débuls de la prise en charge

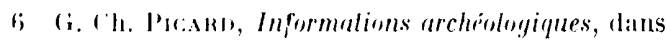
Ciallia, 30, 1972, 1. 32:9. 

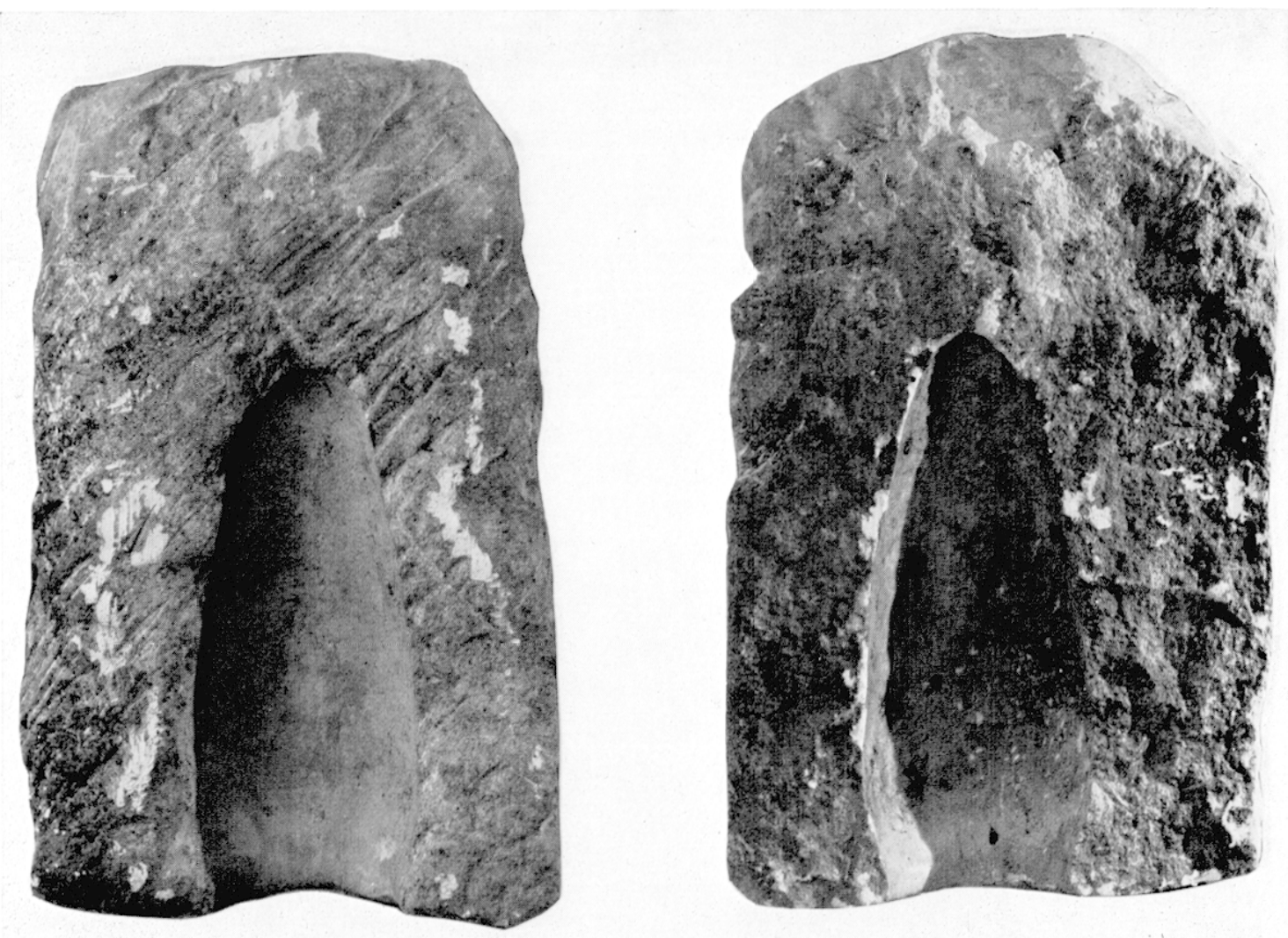

3 La borne de Bellivet : face interne des deux demi-cylindres.

de la vehiculalio sur ces roules par le pouroir impérial?.

On ne peut guère restituer le chiftre manquant pour les distances en considérant le lieu de la trouvaille de la borne. en admetlant ${ }^{8}$ qu'elle ait été découverte à son emplacement d'origine ou à proximité de celui-ci (fig. 1 et 5). Il faudrait en effet connaîlre l'emplarement. exact du capul viarum de Mediolanum. (On pourrait songer ici a l'arc de Germanicus, en référence à une inscription de Cordoue ${ }^{9}$ et en prenant en compte la certitude où nous

7 Voir H.-G. Praci, les procuraleurs équestres sous le Haut-Empire romain, Paris, 1950, p. 87; G. Walser, op. cit., p. 100-103; I. Mauris, saintes Antiques, Saintes, 1978, p. 301 it n. 14.

8 Comme nous te faisons ici, en considerant la présence des deux moilies de la partie superieure de la borne réunies au même endroit, et le fait guu'aucun sarcophage fou autre vestige archoologique n'a ite decouvert en ces lipux.

9 CilL, II, 1721. sommes de son ròle de capul viae vers Lyon. On pourrait songer aussi au carrefour des deux voies principales de Mediolanum (ou au voisinage de ce carrefour), non loin du croisement des cours Reverseaux el National (fig. o). l)ans l'un ou l'autre cas on obtient, à partir de l'endroil de la découverte de la borne, une distance voisine de $4,500 \mathrm{~km}$, ce qui équivaut approximativement aussi bien a trois milles romains $(1481,50 \mathrm{~m} \times 3=4444,50 \mathrm{~m})$ qu'à deux lieues gauloises de $2222,25 \mathrm{~m}$, puisque la lieue valait en principe un mille et demi ${ }^{10}$.

lin aulre intérêt de la borne de Bellivet est de soulever à nouveau la question d'une liaison routiere entre saintes et l'Océan.

10 Éfuivalence donnée surtout par deux auteurs latins lardifs, Ammien Varcellin seconde moitié du IVe siicle; of Jordanes (milie'u du vie siecle); voir Drsonanoss, Géographie de la ciaule Romaine, t. IV, p. $23 \mathrm{~s}$. 


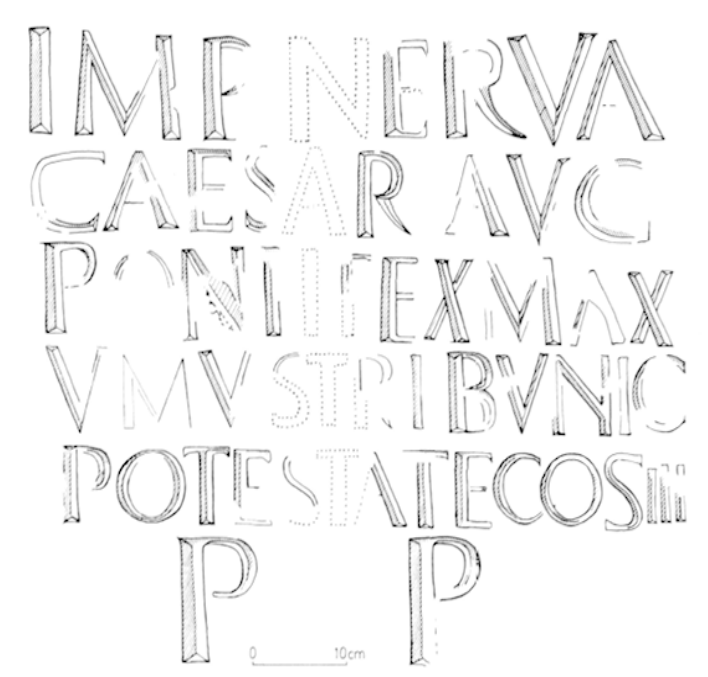

1 Fac-simile de l'inscription.

Jusqu'à présent, on connaissait bien un écheveau de voies anciennes reliant Saintes a la mer, mais, de toute évidence, le réseau routier des grandes voies impériales passait loin des còtes, ne marquant aucun intérêt pour le littoral'11, si l'on veut bien excepter le problème toujours controversé de Tamnum et de Novioregum ${ }^{\mathbf{1 2}}$.

Or la découverte de Bellivel prouve que la sollicitude de l'administration impériale s'adressait également à cette région littorale. Mais il faudrait établir précisément l'itinéraire suivi par la route romaine que notre document révèle. Nous l'avons dit, il y a tout lieu de

11 Sur le réseau routier de l'Antiquité dans cette région, voir notamment : A.-F. I.ACLRIF, Nolice sur le pays des Sanlones à l'époque gallo-romaine, Saintes, 1844 , p. 32 ; P. ol P'. Bt.raAtr, La Vallée, essai d'analyse d'un lerroir rural en Sainlonge, dans Roccaforlis, 2e ser. I, 1958-1964, p. 98-103; 11. Ci.octr, Noles sur les voies romaines parlanl de Mediolanum Sanlonum (Sainles), dans Rev. générale du Centre-Ouest, 36, 1934, p. 212-215; C. GABLT, I, habilat rural gallo-romain enlre la basse Charente el la seudre, dans Cellicum IX, 1963, p. 240. J. I Assif:, Archéologie aérienne en Charenle-Marilime, dans Kev. archéol., 1977, p. 283-306 (on collaboration avec R. Chevallier, P. Delauné, I. Guy, A. Kermorvant, conclusions reprises par J. 1)Assí, Manuel d'archóologie aérienne, Paris, 1978, p. 181-186. L. Matnis, op, cil., p. 299-306.

12 Sur cette question, voir surtout f; Jurl.tax, op. cil., p. 226-23:2.

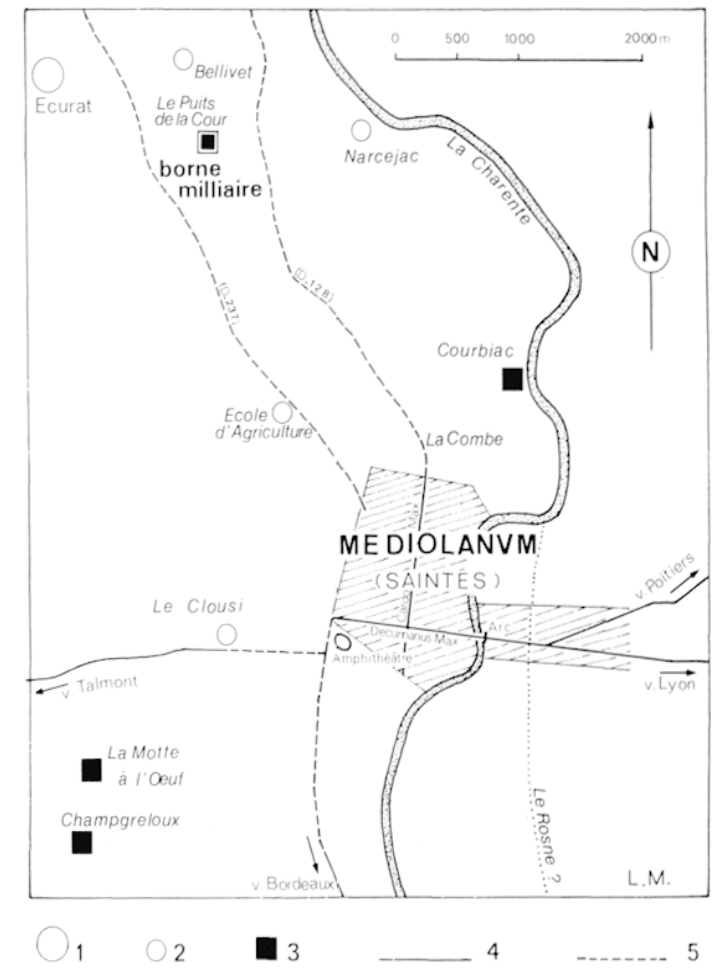

5) Situation de la borne par rapport à Mediolanum : 1, commune; 2 , lieux-dits ; 3 , vestiges de l'epoque galloromaine; 4 , voies romaines assurées; 5 , roies anciennes.

supposer que la borne se trouvait quasiment en place avant son exhumation en 1956 . Cependant les travaux des viographes saintongeais proposent plusieurs solutions pour relier Mediolanum à cette partie nord-ouest de la cité des Santons. Une voie romaine, et même une série de voies ont été décelées ou supposées dans la région de la basse Charente ${ }^{13}$. Des témoignages assurés sur la géographie routière ancienne de cette contrée sont réunis sur la figure 6. L'accumulation et la distribution des habitats antiques reconnus dénonce sans conteste la présence d'un réseau routier, mais l'écheveau des voies anciennes, qui ne sont pas forcément gallo-romaines, proposé par l'interprétation aérienne de la carte au $1 / 50.000 \mathrm{e}$ (donnée ici par J. Soyer), rend les tracés incertains, comme on le constate.

13 F. Eygix, Informalions archéologiques, dans Gallia, XIX, 1961, p. 424-426, et les études citées n. 11 . 


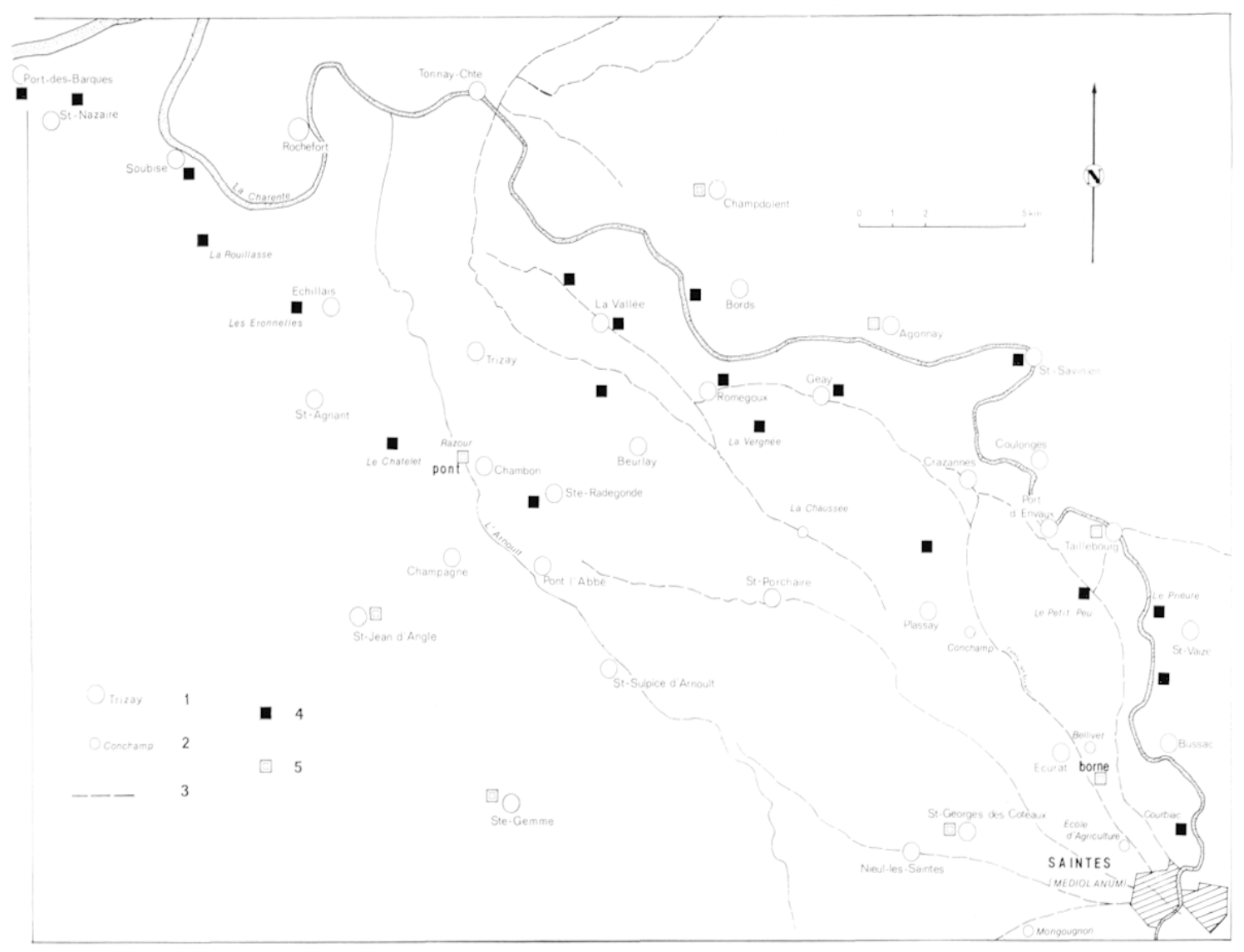

6 La trouvaille de Bellivet al l'occupation du sol à l'eporge gallo-romaine dans la région de la Basse Charente: 1, communes; 2, lienx-dits; 3, voies anciennes reconnues par la photographie aerienne ; f, habitats d'eporgue galloromaine; j, autres vestiges d'époque gallo-romaine.

La borne de Bellivet pouvait se dresser : 10 Sur une roule de Saintes a Tonnay-Charente où elle traversait le fleuve, par la Combe des Jevisions et Conchamp, ou bien par Port d'Envaux, puis Romegoux et La Vallée (où $P$. et P. Burgaud l'auraient reconnue au Gué de Port-Coutard). $2^{0}$ sur une route de saintes a Port-des-Barques, par Plassay, Saint-Porchaire, Pont-l'Abbé d'Arnoult. Razour (commune de Champagne). Son tracé, non attesté archéologiquement, est dénoncé ensuite par les habitats importants du Chatelet, des Eronnelles (commune de saint-Agnant) de la Rouillasse (commune de Soubise) et de Soubise ${ }^{14}$. Ce dernier ilinéraire, proposé par

14 Sur l'occupation du sol a l'aporpue gallo-romaine: dans celte région, voir C. ('ABBET, L'habitat gallo-romain entre la Basse Charenle el la seudre, dans Cellicum IX,
C. Ciabel (suivi par F. Eygun). al l'avantage d'être en outre matérialisé par un ouvrage d'art, le pont de Razour. jeté sur l'Arnoult. Ce pont de bois, ancré sur des culées de pierre, a été retiré des vases de l'Arnoult en 1960), en mème temps que l'on metlait au jour un tronçon de la voie qui $y$ accédait ${ }^{15}$. Mais l'époque, présumée antique, de ce pont, n'est pas parfaitement assurée.

Quelles que soient les incertiludes qui demeurent, un point est arquis: le gouverne-

1963, p. 2.10. Sur l'ensemble du Chatelet (sanctuaire ?', voir P. INAvin et C. GABET, I.e sile gallo-romain du Chalelel, Rochefort, 1973.

15 F. Frot: op cit., p. t25. Eipoque lixée par le chiffre VII gravé sur des poutres de chêne retirés de la vase et par la maconnerie des culés. 
ment imperial s'est préoccupé. au moins depuis

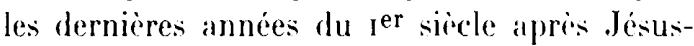
Chrisl, de creer une voie qui desservail les régions de la basse Charente. el de veiller a son entretien. I l'existene de rette roie répondent la densité des habilats. la prospérilé qui semble avoir régne dans celte contré que l'on songe au tresor de quelque quatre mille deniers ou doubles deniers découvert a Ia Rouillasse: l'importance de premier ordre de certains siles avant tout celui du Chatelet.

Mais res restiges sulfisent-ils a expliquer la présence d'une roule imprériale? I a question posée par la borne de Bellivel n'est point tant celle de l'existence de la voie et de son tracé. qui reste incertain. que relle de son aboutissement qui juslifiait l'attention de l'administration de l'kmpire. Pouvons-nous supposer que celle roule desservait un port des santons? Pour répondre a cetle question. il faurdrait examiner les siles propices a l'installation d'un port, et donc connaitre la configuration exacte de la cote charentaise, qui a considerablement évolué pendant les derniers millenaires ${ }^{16}$. Mis a part les aflleurements calcaires de part et d'aulre de l'embouchure de la Charente. la plus grande partie du rivage est constituée par des marais maritimes, hérilage d'anciens golfes marins colmatés au cours de la transgression flandrienne. Pour tous les anciens historiens de la cole. la solulion est simple : pour comnaître la configuration du rivage romain, il suffit de suive la limile de ces marais, donnée par le contact entre bri flandrien et calcaire : on oblient alors une côte extremement découpée et parsemée d'iles comme le montrait déja Desjardins dans sa Géographie ${ }^{17}$. En fait, la encore nous sommes dans la plus crande perplexite, car il faut tenir compte des deux facteurs de l'évolution littorale, a savoir les varialions du niveau marin et le degré de colmalage ${ }^{18}$. Les progres

16 Calle question de la contignation des robles antirgues de la saintonge est developpere dans

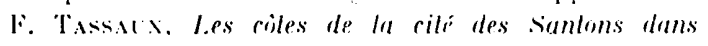
l.Antiquile, dans kelue de la sininlon!fe el de l'. Iunis, I. $1975,1,9-18$.

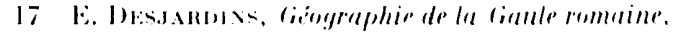
1. I $1 \times 76,10.2633-278$.

Ix II faut y ajouter lerosion des pointes ralcatres. accomplis depuis les quinze dernieres anneses par locéanographie tendent i montrer que le niveau marin actuel a cte atteint peu avant notre ire a I a Tone III / ? 19 . mais les opinions divergent sur le niveau marin de l'époque romane : elait-il égal ou inférieur au niveau actueleb? Lin allendant la réponse que donneront peut-itre les Iravaux des gréloguesese loutes res incertiludes démontrent quil est Ires audacieus pour linstant de rouloir tracer une carte précise du rivage saintongeais a l'epoque romaine el parlant, d'affirmer d'une facon péremptoire, que tel ou lel sile etait impropre ou favorable a l'implantation d'un port ou a la navigation antique.

Les soures lilleraires antiques permettentelles de siluer un queleonque port simton ou aboutirail une vole sianles-()cian? Iceur

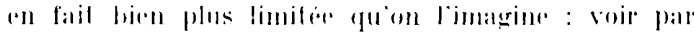

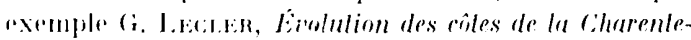
barilime, élude morphorlynamique. Bordanux, 1970, b. 6l Iniversite de Bopdoanx III, lastitut de Georraphie, dacelyl.

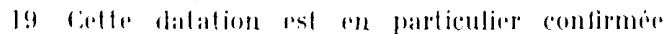
dans la region par la presence de nombrems siles a sel probohistoriques, les uns recouverts par le lor flandrien, les antres installis a la limile de ce bri, voire dessous.

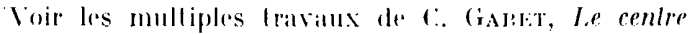
desploitalion du sel de Porl-Coulart, daus Cellicum XII. 196i.1, p. 2:31-2:35; La transqression flandrienne on Alunis ol Sirintonge: nonvelles observations failes alans

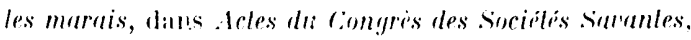
Tours, $196 \times 1970$, 1. 365: Les nariations des lignes du

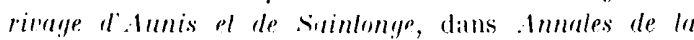
Sincialti des siciences naturelles de la charente Mrarilime, $1,3,1971,1$. 109-123 ; l.e sile a sel de la l'elile dignille, dans Ruccafortis, 2" ser. III, 2, 1973; Le site a sel de

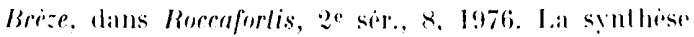
la phus recente sur da funestion de la transerression

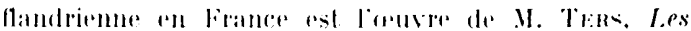
larialions du nirean marin depmis. 10009 ans lo lon!y du lilloral allamlique francais, $X^{\circ}$ congris inlernalional de II.VOC.1, dans le Oualernaire, 1973, p. 11.1-135. Ella

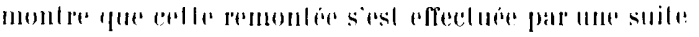

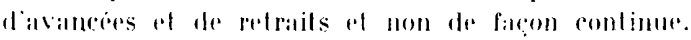

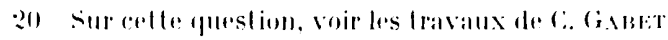
rilis dans la ne procidentre il soppose a un has niveran romain, alors que .I. Trans, Les lignes de ribage hrolocine le lon!l de la crile allanlique francaise, dans La Próhistoire franguise. Paris, 1976, 19. 27-30), conclut à un nivean

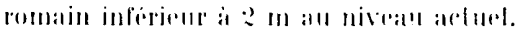

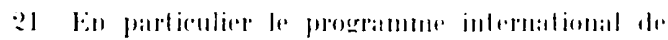

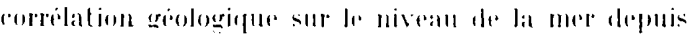

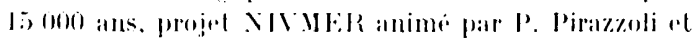
II. Fallur. 
examen est en fait bien décevant ${ }^{22}$. Ia seule indiration vérilablement intéressante est donnée par Ptolémée qui énumère un certain nombre de lieux entre Gironde et loire. avec leurs coordonnées ${ }^{23}$. Depuis l'étude de Berlhelot ${ }^{24}$, nous savons que les coordonnés de Ptolémée ne peuvent être prises a la lettre. mais qu'elles doivent âlre corrigées en fonclion d'une double source d'erreurs inhérentes à la géographie antique. En réalité, tout ce que l'on peut raisonnablement espérer de res chiffres, c'est une estimation tres approximalive de la distance séparant chaque point de l'autre. mais alors leur identification éventuelle doit tenir compte des modifications subies par la cole el des conditions de la navigation antique, sur lesquelles, comme nous l'arons dit, on posside peu de certitudes. Ces données corrigeses sont les suivantes:

lieux donnes $\begin{gathered}\text { Coordonnées Calcul } \\ \text { par Plolémée } \\ \text { de Ptolémée }\end{gathered}$ des
distances

\section{Garouna}

Port des santons. 170:30' 460 $30^{\prime} \quad 5 \%$ km

Caip des Santons.. $160^{\circ} 30^{\prime} \quad 460^{\circ} 45^{\prime} \quad 39 \mathrm{~km}$

Lianenlelos....... $160330^{\prime} \quad 47^{\circ} \quad 15^{\prime} \quad 56 \mathrm{~km}$

Cap des Pictons. $\quad 17^{\circ} \quad 48^{\circ} \quad 24 \mathrm{~km}$

Port Silior....... $170330^{\prime} 48^{\circ} 15^{\prime} \quad 33 \mathrm{~km}$

Ligeir......... $17^{\circ} 40^{\prime} 48^{\circ} 45^{\prime} \quad 22 \mathrm{~km}$

Bien plus que la Charente, la sevre Niortaise fou encore la Vie ou l'Auzance) pourraient.

2.2 Voir I. Malnix, op. cil., p. 271 et. s.

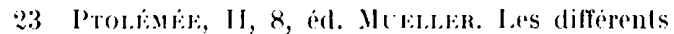
manuscrits de ptolimo ne concordent pas sur les coordonnees du Porl of du calp des santons : on trouve ignlement l'ort des sintons 17030'-17"15', (aip des Santons 16"15'-150.15'. Jans d'aulres mamuscrils, ainsi que che\% Marcien d'Ileraclée, le Port des Santons nesl même pas menlionne; le lap des santons a alors $16^{\circ} 30^{\prime}$ de lompitude of $16^{\circ} .5^{\prime}$ de latilude. Mreater pense que dans le lexte original de Plolemee seul le (a) des Santons etajt indique ot que la Port des Santons a ele rajoule par la suild p. 200 .

2.4 A. Bintunior, La carle de la Gaule de Ptolémie, dans Reo. des Eludes anciennes, 19333, p. 125-435); 1934, p. 293-302: sa demonst ration est adoptere par I.. PAPr, Ia cole allantique de la Iooire à la cirronde, lhomme el la mer, Bordeaux, 1941, p. 1.42. correspondre au Canentelos. I.e Cap des Santons devrait se trouver quelque part sur la côte nord de l'Aunis entre La Rochelle et l'Aiguillon, ou bien a la pointe des Baleines. Quant au Port des Santons, on peut raisonnablement supposer qu'il se trouve entre Maumusson el Ia Rochelle. (On retiendra donc de ce médiocre apport des sources littéraires antiques l'éventualité d'un port au nord ou au sud de l'embouchure de la Charente.

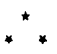

On s'en rapportera done avant tout au témoignage de l'archéologie; on l'a vu, l'embouchure de la Charente, parliculierement la rive sud, porle des traces d'orcupation galloromaine relativement denses, d'Echillais a Port-des-Barques ${ }^{25}$. Celte concentration sur une bande de calcaire assez étroite à l'entrée du seul axe véritablement important de pénétralion fluviale du Centre-()uest. conduisant a la capitale des Santons, pourrail àre un indice sérieux de la présence d'un etablissement portuaire dans les parages. Celte présomption se trouve renforcée par la convergence de plusieurs voies anciennes, dont l'une était une route impériale, comme nous l'indique la borne de Bellivet. Il resterait a découvrir les vestiges d'un éventuel port anlique dans les parages de Soubise ou de Porl-des-Barques.

\section{Louis llackix et Francis Tassaux.}

25) On rotrouve me concentration de vestiges anticques encore plus forte sur l'elroite hande calcaire rolre le marais de Brouage ef l'esluaire de la Sendre, oi l'on me comple pas moins de neuf sites gallo-romains C. Cis Bar, Habilat rural... . Celle densite relativement forte contraste avec le rivage de l'Aunis. Toutefois on ne prut plus parler de desert areheologique dans cette

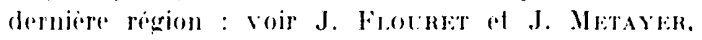
Imenlaire archoologique de liunis, période galloromaine, dans kevue de la saintonge et de l'anis IV, $197 x$, p. $69-7 x$.

N.B. - Tous les dessins sont de Ioujs Maurin dign. $1,4,5$ e 6 , el les clichis du Musée archeologique: de Saintes:fig. 2 at 3 , 